NISSUNA UMANA INVESTIGAZIONE SI PUO DIMANDARE VERA SCIENZIA S'ESSA NON PASSA PER LE MATEMATICHE DIMOSTRAZIONI LEONARDO DA VINCI

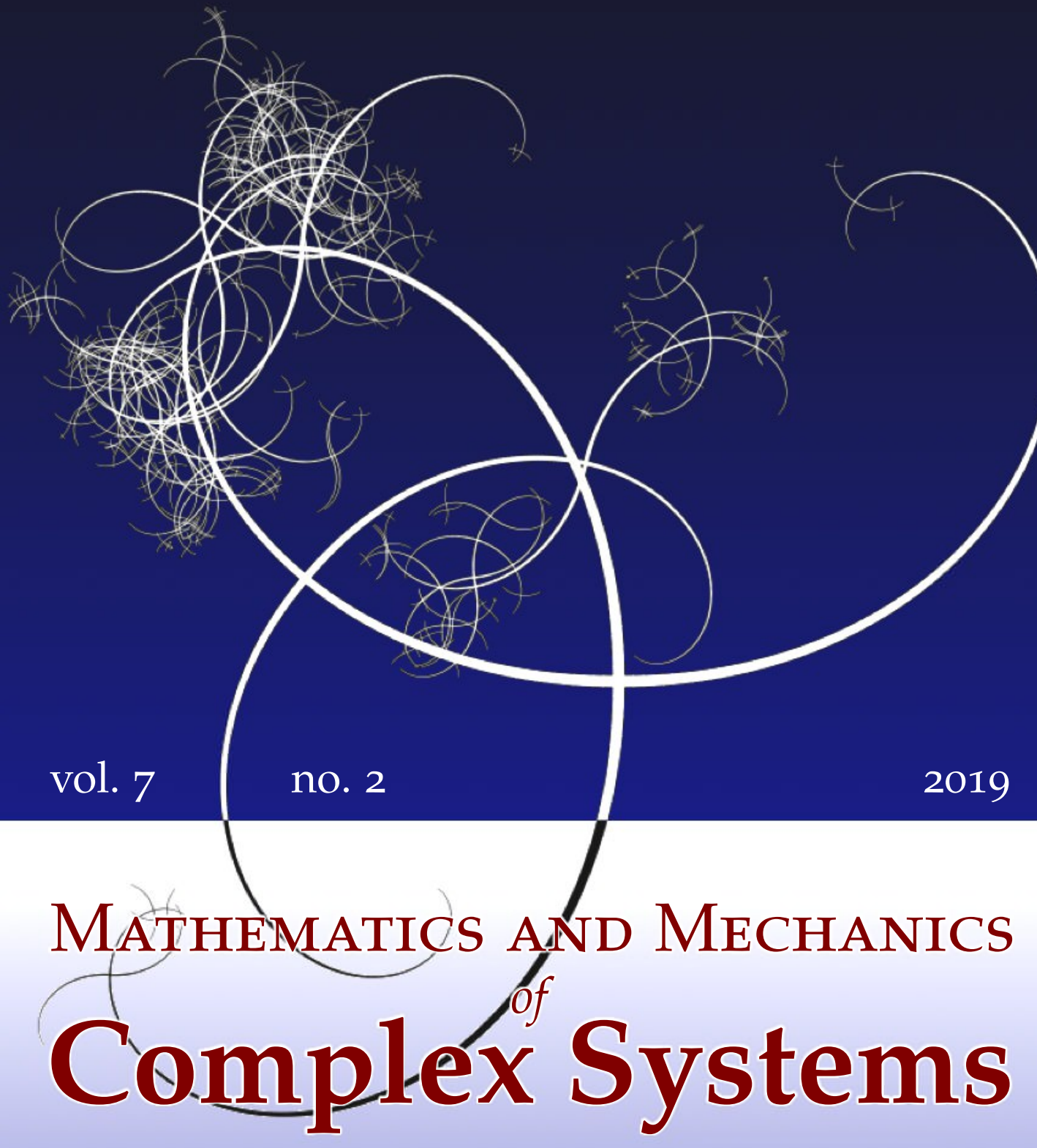

Mikhail A. Guzev, Gurami S. Tsitsiashvili and Marina A. Osipova THE OBJECT DETECTION BY AUTONOMOUS APPARATUS AS A SOLUTION OF THE BUFFON NEEDLE PROBLEM 


\title{
THE OBJECT DETECTION BY AUTONOMOUS APPARATUS AS A SOLUTION OF THE BUFFON NEEDLE PROBLEM
}

\author{
Mikhail A. Guzev, Gurami S. Tsitsiashvili and Marina A. Osipova
}

\begin{abstract}
The problem of object detection by autonomous apparatus is considered. The probabilistic formulation of the problem is proposed by means of a reduction to the classical Buffon problem. The latter naturally arises when the problem is formulated in the coordinate system associated with the apparatus. The problem of detection is considered for devices moving in the open space along a circle around one body, for vehicles patrolling along the linear boundary protecting the bodies, and for devices protecting the system of bodies. The problem of object detection was shown to admit an analysis in the presence of an asymptotic parameter determined by the ratio of the local size of the apparatus scanning area to the global size of the problem under consideration. For all problems, the minimum number of apparatuses that could detect a penetrating object with probability one was calculated.
\end{abstract}

\section{Introduction}

Nowadays, the attention of researchers is focused on the study of self-propelled particle systems [Della Corte et al. 2016; Brambilla et al. 2013; Bellomo and Brezzi 2016; Adamatzky and Jones 2008; Herrero and Soler 2015]. Analysis of these systems leads to the need to solve various problems closely related to the theory of dynamical systems. The simplest systems can be the systems of particles studied in the framework of Maxwell-Boltzmann, Fermi-Dirac, and Bose-Einstein statistics [Kardar 2007]. Billiard systems, where stochastic properties can arise even with a small number of particles and in the absence of random factors [Zaslavsky 2007], are an important analogue of self-propelled particles systems.

When considering the self-propelled particle systems, the number of particles is, as a rule, assumed to be sufficiently large. In particular, such assumptions are used in modeling the movement of vehicles, crowds and flocks, financial markets, and other socio-economic systems [Herrero and Soler 2015], consisting of a sufficiently large number of self-driven particles. In this connection, one should point out

\section{Communicated by Francesco dell'Isola.}

MSC2010: primary 60D05; secondary 70Q05.

Keywords: extraneous mobile object, autonomous unmanned vehicle, detection probability. 


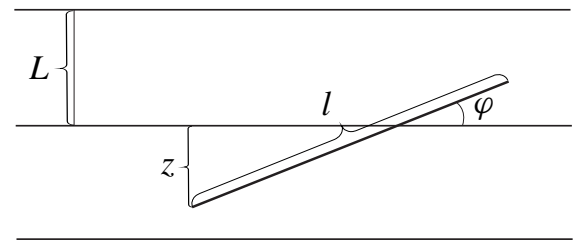

Figure 1. The Buffon needle problem.

the model of the quantum economy [Maslov 2005], in which a large number of self-driven particles leads to the appearance of synergistic effects - the nonlinear (exponential or power) dependence of the efficiency indicators on the number of particles.

At the same time, the self-propelled particle systems are close to the mobile robot systems that can be equipped with artificial intelligence. To describe the dynamics of the mobile robot systems, the models of differential games [Alspach 2004; Chung et al. 2011; Galceran and Carreras 2013], random graph models [Hazra et al. 2018; 2017a; 2017b], which underlie many artificial intelligence systems, etc., are used. Within these model frameworks, different problems are solved: determination of the shortest trajectory of a robot or a group of robots which covers the entire field of vision [Alspach 2004; Galceran and Carreras 2013], searching for the minimum number of robots that guarantee the capture [Chung et al. 2011], and many others.

This paper is devoted to solving the problem of calculating the detection probability of a mobile object penetrating through a system of mobile robots modeled by self-propelled particles and not possessing a sufficiently developed artificial intelligence. Such a problem may arise when designing a system of mobile robots that track the object penetration into a controlled area at sea, on land, in air, etc.

We analyze this problem using the solution of Buffon's problem [Kendall and Moran 1963; Ambartzumian 1982] about calculating the probability of intersection of a random segment on a plane with a system of parallel lines. This model takes into consideration the kinematic and geometric features of the movement of mobile robots and the mobile object much more fully.

The Buffon problem (see Figure 1) is the determination of the probability of intersection of a needle with length $l$, which is randomly thrown onto a plane ruled by equally spaced parallel lines a distance $L$ apart, with any one of these lines. This problem formed the basis for stochastic geometry and was widely used in applied statistics. The main elements of the probabilistic model in the Buffon problem are the random variables that determine the mutual position of the segment, occupied on the plane by the needle, and the equally spaced horizontal lines [Ambartzumian 1982; Kendall and Moran 1963]. The distance $z$ from the segment's lower end to 
the first overlying line and the angle $\varphi<\pi$ between this line and the segment are such random variables. Knowing the distribution law of the random vector $(z, \varphi)$ formed by these random variables, we can determine the probability of the event $P(l \sin \varphi \geq z)$ of the needle intersection with one of the parallel straight lines. For this event to happen, it is necessary and sufficient that the segment intersect with a straight line lying directly above its lower end.

In this paper we tried to adopt the Buffon problem to consider object detection by autonomous apparatus. The article outline is as follows.

In Section 2 we describe the joint motion of the vehicles and the object in order to calculate the probability of the intersection of the trajectory of an object, which is moving toward the body, with the apparatus scanning area. To determine the probability of the object detection, this model is recorded in a coordinate system associated with devices moving along a circle around the protected body.

In Section 3, we performed an analytical study of the proposed model for movement of the vehicles and the object in the polar coordinate system. With a certain ratio between the linear size of the detection area and the size of the area of the devices' motion, this task differs little from the classical Buffon problem. The resulting reduction allows us to calculate the minimum number of vehicles at which the probability of detecting an object is close to one.

Cyclic motion of vehicles along a closed linear segment is analyzed in Section 4. To calculate the minimum number of vehicles for which the probability of the object detection is close to one, the approach proposed in Section 2 is used, which consists of a transition to the coordinate system of the moving device.

In Section 5, we consider the protection model of the network structure of bodies which are located in the nodes of a square lattice bounded by the circle along which the vehicles move. For two different strategies of the vehicles' movement, the minimum number of vehicles was determined at which the probability of the object detection is close to one, and the most effective strategy is indicated.

Section 6 presents the final comments on the study performed and identifies the statements of new problems that follow from the considerations made in this paper.

Material of the article is partially presented in [Guzev et al. 2018], which examines the vehicle's movement along a circle around a single protected body and along a straight line segment.

\section{Buffon problem in the coordinate system associated with vehicles moving around a circle}

Let $n$ vehicles move along a circle of radius $R$ with a fixed linear velocity $v$ and at an equal distance from each other. Each of these vehicles is equipped with circular radar with scanning radius $r$ (see Figure 2). We will use the following symbols 


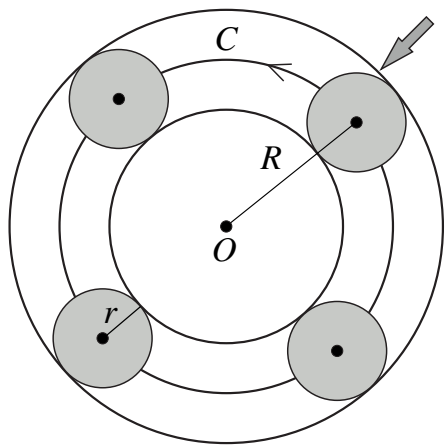

Figure 2. The vehicles' movement around a circle in a stationary coordinate system.

in all drawings: black dots are protected bodies, black arrows show the direction of vehicle motion, filled circles are the area of the locator survey, and wide filled arrows show the direction of object motion.

The circular radar can be understood as a vehicle that rotates a beam with an angular velocity large enough for the linear velocity of the beam to notably exceed the linear speed of the vehicle.

It is required to calculate the probability of detection of a mobile object by means of locators installed on the vehicles. By detection, we mean here that the object falls into a circle which is scanned by any of the vehicles.

The peculiarity of the task of calculating the probability of detecting a mobile object in a stationary coordinate system, associated with an external observer, is that both the vehicle and the object are moving, and therefore, some transition to the Buffon problem, in which at least parallel straight lines are fixed, is required.

Such a transition is realized when the motion of the object under study is considered in a coordinate system associated with vehicles rotating around a certain point $O$ along a circle $C$ of radius $R$. In this coordinate system, the radar scan circles with radius $r$ become fixed. To be detected by the vehicle, the object trajectory should intersect one of these circles.

In order to simplify the study of this problem, we suppose that, in a stationary coordinate system, the mobile object moves with the velocity $u$ along a segment connecting the starting point of its movement with the center $O$ of the circle $C$.

In a rotating coordinate system, the shape of the object trajectory $A_{\psi}$ differs from the segment (see Figure 3): it will be some curve that starts at a random point $\psi$ of the circle $C_{*}$, with radius $R+r$ and with center at $O$. Thus, in the coordinate system associated with the orbiting vehicles, circles of radius $r$ play the role of parallel lines and curves $A_{\psi}$ the role of the segments in the problem of Buffon.

We further assume everywhere in this paper that the random angle $\psi$ has a uniform distribution on the segment $[0,2 \pi]$. The transition from the curve $A_{\psi^{\prime}}$ 


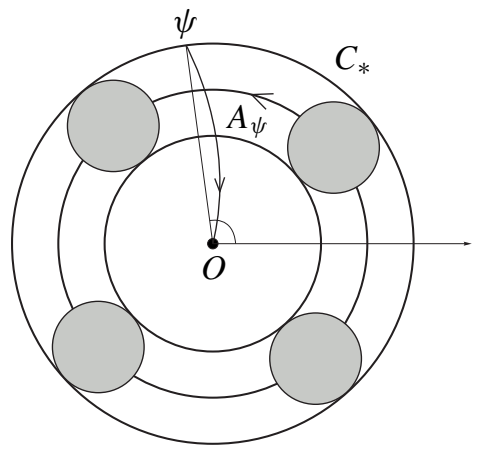

Figure 3. Trajectory of object motion in a Cartesian coordinate system associated with vehicles moving around a circle.

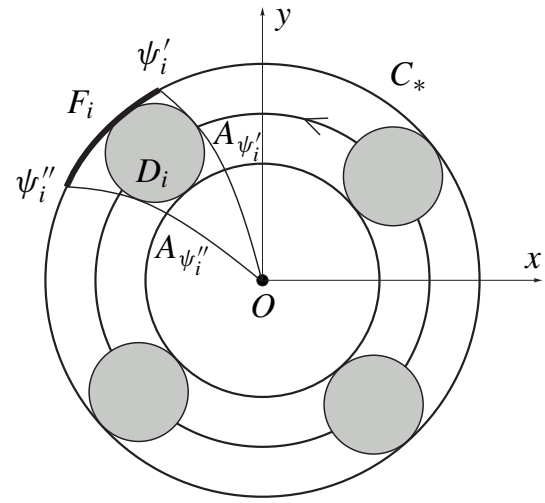

Figure 4. Control area of one circle of radar scan in the Cartesian coordinate system $(x, y)$ associated with moving vehicles.

to the curve $A_{\psi^{\prime \prime}}$ is carried out by rotation of the curve $A_{\psi^{\prime}}$ around the point $O$ by the angle $\psi^{\prime \prime}-\psi^{\prime}$. It should be noted that, on the circle $C_{*}$, each circle $D_{i}$ with radius $r$ allocates an arc $F_{i}$ bounded by two curves $A_{\psi_{i}^{\prime}}$ and $A_{\psi_{i}^{\prime \prime}}$ that touch the circle $D_{i}, i=l, \ldots, n$, from the outside (see Figure 4). Thus, on the circle $C_{*}$, the scan circle $D_{i}$ with the center at the point, where the apparatus $i$ is located, allocates the arc $F_{i}, i=1, \ldots, n$. Now we can calculate the probability of detection of a mobile object by circular view radars installed on vehicles. Let the set $\mathcal{F}$ be the union of the $\operatorname{arcs} F_{i}: \mathcal{F}=\bigcup_{i=1}^{n} F_{i}$. This set is the union of a finite number of disjoint arcs (which may be less than $n$ ). Then the total angular length $s(\mathcal{F})$ of those arcs divided by $2 \pi$ is the required probability of detection a mobile object by radar locators installed on the vehicles.

Therefore, if the $\operatorname{arcs} F_{i}, i=1, \ldots, n$, do not intersect, the probability of the object detection by the vehicles is $P=n s\left(F_{i}\right) /(2 \pi)$, where $s\left(F_{i}\right)$ is the angular length of the arc $F_{i}$. In the general case, $P=\min \left(1, n s\left(F_{i}\right) /(2 \pi)\right)$. 


\section{Buffon problem in the polar coordinate system}

The task of determining the curves $A_{\psi}$ and finding the $\operatorname{arcs} F_{i}$ is solved, as a rule, numerically. However, at some additional assumptions, it is possible to obtain analytical formulas. Let us transition from the rectangular coordinate system $(x, y)$ associated with the vehicle rotating around point $O$ to the polar coordinate system (see Figure 5, left) with the vertical coordinate $\rho=\sqrt{x^{2}+y^{2}}$ and the horizontal coordinate $\varphi=\arctan (x / y)$.

In a rectangular coordinate system $(x, y)$, a circle of radius $r$ centered at $(O, R)$ is described by equations

$$
x=x(\psi)=r \cos \psi, \quad y=y(\psi)=R+r \sin \psi, \quad 0 \leq \psi \leq 2 \pi,
$$

giving a parametric definition of the circle: $\psi$ is a parameter. In the polar coordinate system $(\rho, \varphi)$ this circle is given parametrically by the equations

$$
\frac{\rho}{R}=\left(\frac{r^{2}}{R^{2}} \cos ^{2} \psi+\left(1+\frac{r}{R} \sin \psi\right)^{2}\right)^{1 / 2}, \quad \varphi=\arctan \frac{(r / R) \cos \psi}{1+(r / R) \sin \psi},
$$

since $\psi$ remains a parameter. A typical image of a circle with a radius $r$ in this polar coordinate system looks like an oval with pointed upper end (see Figure 5, left). For small values of the parameter $r / R \ll 1$, the last system of equations can be approximated as

$$
\frac{\rho}{R} \approx 1+\frac{r}{R} \sin \psi, \quad \varphi \approx \frac{r}{R} \cos \psi
$$

Thus, a circle of radius $r$ in the coordinate system $(x, y)$, rotating with the apparatus, transforms into almost a circle of radius $r / R$ in a normalized polar coordinate system $(\rho / R, \varphi)$ (see Figure 5, right). The linear speed of the vehicle is $v$, and
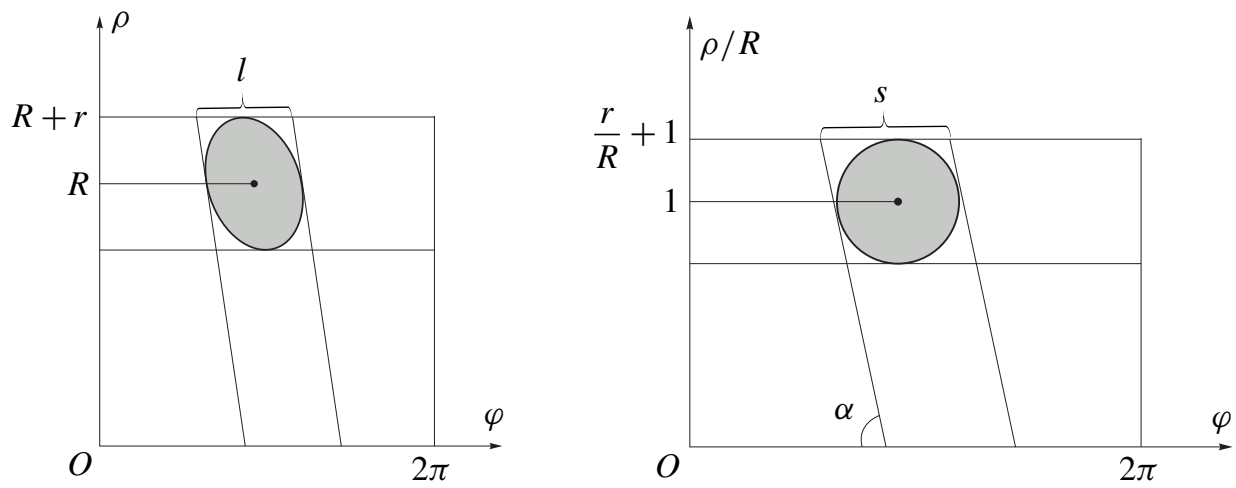

Figure 5. The control area of one circle of radar scan in the polar coordinate system (left) and in the normalized polar coordinate (right) associated with moving vehicles. 
the speed of the mobile object in the fixed coordinate system is $u$, so in a fixed Cartesian coordinate system, the object moves to the center $O$ of the circle $C_{*}$ with velocity $v$, and the vehicles move along the circle $C$ (in the angle $\varphi$ ) with the angular velocity $u / R$. In the fixed polar coordinate system $(\rho / R, \varphi)$, the object moves along the radial coordinate $\rho / R$ with velocity $v / R$, and the vehicles move along the angular coordinate $\varphi$ with velocity $u / R$. Therefore, in the polar coordinate system $(\rho / R, \varphi)$ associated with the vehicles, the vehicles are fixed, whereas the object moves along the radial coordinate $\rho / R$ with the velocity $v / R$, and along the angular coordinate $\varphi$ with velocity $-u / R$ [Dreizler and Lüdde 2010].

Then, in the normalized polar coordinate system $(\rho / R, \varphi)$ associated with the moving vehicles, the speed of the mobile object is equal to $\sqrt{v^{2}+u^{2}} / R$ and makes an angle $\alpha=\arctan (u / v)$ with line $\rho / R=0$. Therefore, each circle representing the control area of the radar locator overlaps a segment of length $s=2 r /(R \sin \alpha)$ for the mobile object (see Figure 5, right).

Let's move from the probability of the object detection to the minimum number of vehicles $M=\min (n: n s \geq 2 \pi)$ at which the probability of a mobile object detection is equal to one; then

$$
M=\min (n: n r \geq R \sin \alpha), \quad \frac{R \sin \alpha}{r} \leq M \leq \frac{R \sin \alpha}{r}+1 .
$$

If the number of vehicles is $n=M$, then the distance between the centers of the neighboring survey circles can obviously be less than $l$.

\section{The movement of vehicles along a straight line}

Now let the vehicles move along a segment $B A$ of length $L$ in one direction and, after reaching its end, turn and move in the opposite direction (see Figure 6). We believe that the distance between neighboring vehicles is $2 L / n$; a similar model was considered in [Kozhemyakin et al. 2017].

Then the scan circles associated with the vehicle locators move along the cylindrical surface with the generatrix $A_{*} A_{* *}=B_{*} B_{* *}$ of length $2 r$ and the directrix

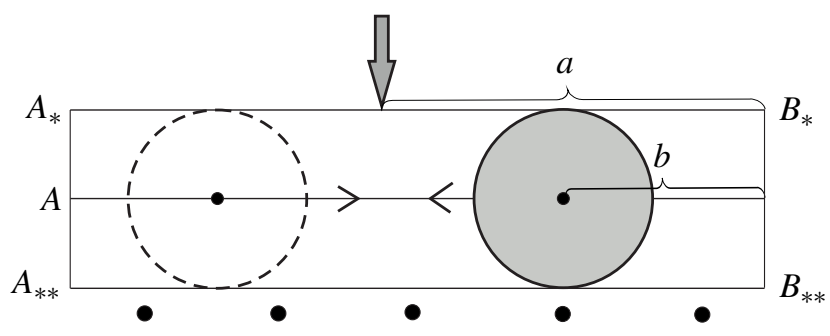

Figure 6. Vehicle and object motion in a fixed coordinate system.

The bold arrow indicates the direction the object is moving. 


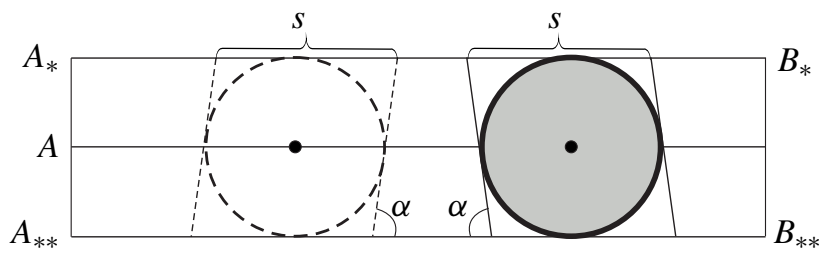

Figure 7. Vehicle and object motion in a coordinate system associated with the vehicles moving along the cylindrical surface.

$B_{*} A_{*} B_{*}$ of length $2 L$. In a fixed coordinate system, the object moves vertically, and its horizontal coordinate at the time of crossing the strip is $a, 0 \leq a \leq L$. Let's transition to the coordinate system associated with the vehicles moving along the cylindrical surface and denote as $b$ the distance from point $B$ to the nearest vehicle in the movement direction (from right to left in Figure 6).

Let the probabilistic distribution of the random vector $(a, b)$ be uniform on the rectangle $[0, L] \times[0,2 L / n]$. Then, in the coordinate system associated with moving vehicles, we can specify subsegments of length $l=2 \mathrm{r} / \sin \alpha$ on the segment $A_{*} B_{*}$, where the object can be detected (see Figure 7).

The task of calculating the probability of the object detection is more complicated when the vehicles are moving along a straight line but not along a circle. A relatively simple solution to this problem was obtained by transitioning from the probability of the object detection to the minimum number $M$ of apparatuses for which the probability of the object detection is equal to one, namely $M=\min (n: n r \geq L \sin \alpha)$. Such a technique can be applied for multiagent systems, considered in [Maggio et al. 2013].

\section{Protection of the net structure}

The network structure consists of bodies in the nodes of a square lattice located inside a large circle. To protect the network structure, the following strategies can be proposed. The first strategy involves the protection of each body separately (see Figure 8 , left). Let $M_{1}(n)$ be the minimum number of devices that implement this strategy. However, we are trying to choose a defense strategy that reduces the number of devices. Therefore, we propose a second strategy to protect the entire network structure (see Figure 8, right). The minimum number of machines implementing the second strategy is denoted by $M_{2}(n)$. The calculation of $M_{1}(n)$ and $M_{2}(n)$ is based on formula (1), which determines the minimum number of vehicles moving around the body at the center of the circle.

For convenience in describing various ways to protect the network structure of bodies from object penetration, we transition from a Cartesian coordinate system $(x, y)$ to a system of dimensionless coordinates $(X, Y), X=x /(2(r+R))$ and 

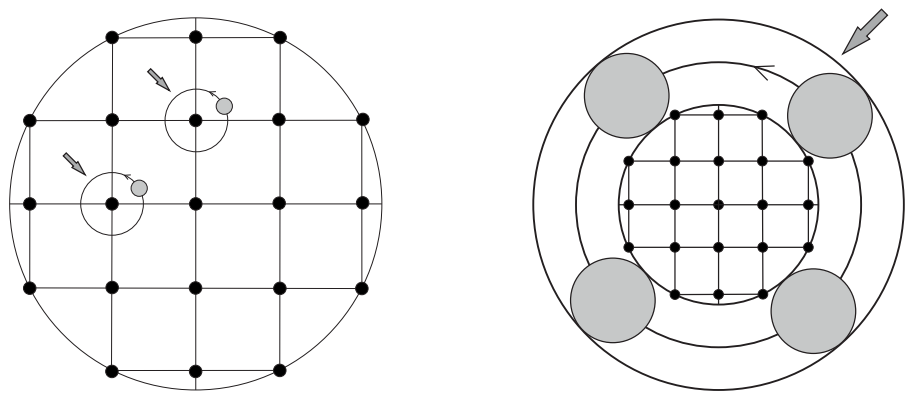

Figure 8. Strategies of apparatus motion: first (left) and second (right).

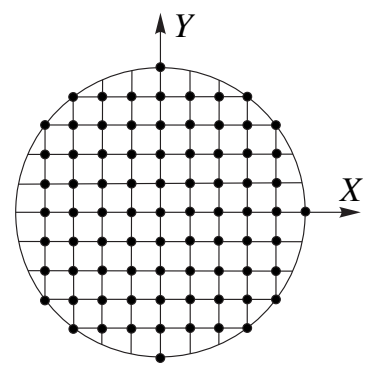

Figure 9. The location of bodies in the network structure.

$Y=y /(2(r+R))$. The parameters $r$ and $R$ were defined earlier in Section 2 . In this coordinate system, a circle of radius $r+R$ is transformed into a circle of radius $\frac{1}{2}$. In the coordinate system $(X, Y)$, consider a circle $C_{n}$ of radius $n$ centered at the coordinate origin and an integer square lattice (see Figure 9). Suppose that there are bodies in the nodes of this lattice inside the circle $C_{n}$, and $N(n)$ denotes the number of these bodies. In the original coordinate system $(x, y)$, the circle $C_{n}$ has a radius $T_{n}=2(r+R) n$.

Around each of these bodies, its own group of vehicles moves along a circle of radius $R /(r 2(r+R))$; in the coordinate system $(x, y)$, this circle has radius $R$. Obviously, these circles do not intersect each other and lie entirely in a circle of radius $n+1$. Then, in accordance with (1), $M_{1}(n) \sim N(n), N(n) \gg 1$, vehicles are needed to find with probability one an object that can move to any of these $N(n)$ bodies, and by virtue of (1),

$$
N(n) f \leq M_{1}(n) \leq N(n)(f+1), \quad f=\frac{R \sin \alpha}{r} .
$$

Consider an alternative protection system for $N(n)$ bodies, assuming that the vehicles move along a circle with a center at $(0,0)$ and a radius of $n+1$; in the $(x, y)$ coordinate system, the radius of this circle is $2(n+1)(r+R)$. Then the 
minimum number of vehicles $M_{2}(n)$ moving along a circle of radius $2(n+1)(R+r)$ (in the coordinate system $(x, y))$ satisfies the relations

$$
2(n+1) g \leq M_{2}(n) \leq 2(n+1) g+1, \quad g=\frac{(R+r) \sin \alpha}{r} .
$$

We now use the known estimates for $N(n)$. So in [Hardy 1999] an estimate of Gauss is given, showing that the number $N(n)$ is equal to the area of the figure composed of unit squares of the lattice, for which the lower left corner lies in the circle $C_{n}$. Since the greatest distance between the points of the unit square does not exceed $\sqrt{2}$, all unit squares that intersect the boundary of the circle $C_{n}$ are located in the ring

$$
\left\{(X, Y):(n-\sqrt{2})^{2} \leq X^{2}+Y^{2} \leq(n+\sqrt{2})^{2}\right\}
$$

with area $4 \pi \sqrt{2} n$. Therefore, relations

$$
\left|\frac{N(n)}{\pi n^{2}}-1\right| \leq \frac{4 \sqrt{2}}{n} \quad \Rightarrow \quad N(n) \sim \pi n^{2}, \quad n \gg 1
$$

hold.

Thus, $M_{1}(n) \sim N(n) \sim n^{2}$ and $M_{2}(n) \sim n, n \gg 1$ (it follows from (3) and (4)). Combining relations (2)-(4), we obtain that for $n \gg 1$ estimates $M_{1}(n) / M_{2}(n) \sim n$ are valid.

Consequently, the application of the protection of the body system reduces the number of vehicles in proportion to $n \gg 1$ compared to the protection of each body separately. The value of $n$ is equal to the radius $T_{n}$ of the large circle which surrounds the lattice points, divided by the lattice step $2(r+R)$.

The estimation of the $N(n)$ value, given in (4), was repeatedly refined. Thus, Gauss showed that $\left|N(10) /\left(\pi \cdot 10^{2}\right)-1\right| \leq \frac{1}{100}$, whereas for $n=10$ the righthand side of inequality (4) is approximately equal to $\frac{566}{1000}$. The refinement of the estimation of the $N(n)$ value [Hardy 1915; Huxley 2002] continues nowadays and is one of the key problems of analytic number theory.

It should be emphasized in conclusion that we considered a dynamic version of the Buffon problem which includes such characteristics as the trajectories and speeds of the mobile extraneous object and unmanned underwater vehicles. This circumstance leads to the necessity of transitioning to coordinate systems associated with moving vehicles and to additional, not always obvious, geometric constructions and the application of the small parameter method.

In addition to the trajectory of the object movement to the center $O$ of circle $C$ (see Figure 2) along the radius, other trajectories are possible, for example, helically [Guzev et al. 2017]. In this case, the body is protected if the viewing circle of each locator intersects with the viewing circle of another locator (see Figure 10). The 


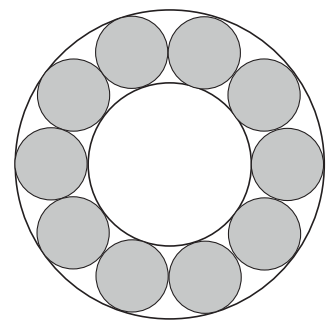

Figure 10. The disposition of the viewing circles of the locators to control the object movement along an arbitrary trajectory.

minimum number of devices detecting an object with probability one is $M^{\prime}=$ $\min \left(n: n s^{\prime} \geq 2 \pi\right), s^{\prime}=2 d$ and $d=\arcsin (r / R)$, and hence $\pi / d \leq M^{\prime} \leq \pi / d+1$.

Let $M_{1}^{\prime}(n)$ be the minimum number of vehicles that provide the detection of the object arbitrarily moving towards each body in the specified system; then

$$
N(n) \frac{\pi}{d} \leq M_{1}^{\prime}(n) \leq N(n)\left(\frac{\pi}{d}+1\right) .
$$

Let $M_{2}^{\prime}(n)$ be the minimal number of vehicles moving along a circle of radius $2(n+1)(R+r)$ and providing detection of an object in its arbitrary moving; then

$$
\pi\left(\arcsin \frac{r}{2(n+1)(R+r)}\right)^{-1} \leq M_{2}^{\prime}(n) \leq \pi\left(\arcsin \frac{r}{2(n+1)(R+r)}\right)^{-1}+1 .
$$

From (4)-(6) we obtain that, in the case of an object with arbitrary motion, the protection of a system of bodies also requires a smaller number of vehicles than the protection of each body separately. The decrease in the vehicle number is proportional to $n \gg 1$, equal to the radius $T_{n}$ divided by the step length of the lattice $2(r+R): M_{1}^{\prime}(n) / M_{2}^{\prime}(n) \sim n$.

\section{Conclusions}

In searching for a solution to the problem of detecting the object, which is moving to the protected body, we came to the classical Buffon model. With such a reduction, obtaining the corresponding analytical results of the original problem turned out to be possible, provided that the vehicles move along a circle around the protected body. The constructed solution was used to analyze the possibilities of protecting the network structure of the body system. Note that the application of the results obtained for a single body to the body system became possible due to the homogeneity of the internal structure of the system and to the preservation of the geometry of the original problem at the system boundary. In particular, such a structure homogeneity is fixed by choosing the same scales in two orthogonal directions, and the geometry of the boundary is given by a circle. 
The generalization of the results obtained is possible if we expand the set of the vehicles' trajectories. For engineering applications, the use of the zigzag motions is of interest [Galceran and Carreras 2013; Tuphanov and Scherbatyuk 2015], the effectiveness of which is confirmed in practice. However, an understanding of the fundamental aspects of the choice of control parameters for such a strategy is a blind spot of modern model theory. Therefore, gaining new knowledge for researchers engaged in this field is undoubtedly relevant.

\section{Acknowledgments}

The authors are grateful to corresponding member of Russian Academy of Sciences A. F. Shcherbatyuk for useful discussions.

This work was partially supported by the Far Eastern Branch of the Russian Academy of Sciences (program "Far East" projects 18-5-050 and 18-5-083) and by the Russian Foundation for Basic Research (project 17-07-00177).

\section{References}

[Adamatzky and Jones 2008] A. Adamatzky and J. Jones, “Towards Physarum robots: computing and manipulating on water surface”, J. Bionic Eng. 5:4 (2008), 348-357.

[Alspach 2004] B. Alspach, "Searching and sweeping graphs: a brief survey", Matematiche (Catania) 59:1-2 (2004), 5-37.

[Ambartzumian 1982] R. V. Ambartzumian, Combinatorial integral geometry: with applications to mathematical stereology, Wiley, 1982.

[Bellomo and Brezzi 2016] N. Bellomo and F. Brezzi, "Mathematics, complexity and multiscale features of large systems of self-propelled particles", Math. Models Methods Appl. Sci. 26:2 (2016), 207-214.

[Brambilla et al. 2013] M. Brambilla, E. Ferrante, M. Birattari, and M. Dorigo, "Swarm robotics: a review from the swarm engineering perspective", Swarm Intell. 7:1 (2013), 1-41.

[Chung et al. 2011] T. H. Chung, G. A. Hollinger, and V. Isler, "Search and pursuit-evasion in mobile robotics", Auton. Robot. 31:4 (2011), 299-316.

[Della Corte et al. 2016] A. Della Corte, A. Battista, and F. dell'Isola, "Referential description of the evolution of a 2D swarm of robots interacting with the closer neighbors: perspectives of continuum modeling via higher gradient continua", Int. J. Nonlin. Mech. 80 (2016), 209-220.

[Dreizler and Lüdde 2010] R. M. Dreizler and C. S. Lüdde, Theoretical mechanics: theoretical physics, 1, Springer, 2010.

[Galceran and Carreras 2013] E. Galceran and M. Carreras, "A survey on coverage path planning for robotics", Robot. Auton. Syst. 61:12 (2013), 1258-1276.

[Guzev et al. 2017] M. A. Guzev, G. Sh. Tsitsiashvili, M. A. Osipova, and M. S. Sporyshev, "Probability of detection of an extraneous mobile object by autonomous unmanned underwater vehicles", pp. 426-432 in 7th All-Russian Scientific and Technical Conference "Technical Problems of the Development of the World Ocean" (Vladivostok, Russia, 2017), Institute for Problems of Marine Technologies, Vladivostok, Russia, 2017. In Russian. 
[Guzev et al. 2018] M. A. Guzev, G. Sh. Tsitsiashvili, M. A. Osipova, and M. S. Sporyshev, "Probability of detection of an extraneous mobile object by autonomous unmanned underwater vehicles as a solution of the Buffon problem", preprint, 2018. arXiv

[Hardy 1915] G. H. Hardy, "On the expression of a number as the sum of two squares", Quart. J. Math. 46 (1915), 263-283.

[Hardy 1999] G. H. Hardy, Ramanujan: twelve lectures on subjects suggested by his life and work, 3rd ed., American Mathematical Society, Providence, RI, 1999.

[Hazra et al. 2017a] T. Hazra, C. R. S. Kumar, and M. Nene, "Multi-agent target searching with time constraints using game-theoretic approaches", Kybernetes 46:8 (2017), 1278-1302.

[Hazra et al. 2017b] T. Hazra, M. J. Nene, and C. R. S. Kumar, "A strategic framework for searching mobile targets using mobile sensors”, Wireless Pers. Commun. 95:4 (2017), 4681-4696.

[Hazra et al. 2018] T. Hazra, M. J. Nene, and C. R. S. Kumar, "Modelling and analysis of informationbased target searching using mobile sensors", Int. J. Sys. Cont. Commun. 9:1 (2018), 53-74.

[Herrero and Soler 2015] M. A. Herrero and J. Soler, "Cooperation, competition, organization: the dynamics of interacting living populations”, Math. Models Methods Appl. Sci. 25:13 (2015), 24072415 .

[Huxley 2002] M. N. Huxley, "Integer points, exponential sums and the Riemann zeta function", pp. 275-290 in Number theory for the millennium, II (Urbana, IL, 2000), edited by M. A. Bennett et al., Peters, Natick, MA, 2002.

[Kardar 2007] M. Kardar, Statistical physics of fields, Cambridge University, 2007.

[Kendall and Moran 1963] M. G. Kendall and P. A. P. Moran, Geometrical probability, Griffin's Statistical Monographs \& Courses 10, Hafner, New York, 1963.

[Kozhemyakin et al. 2017] I. V. Kozhemyakin, D. V. Nikishenko, V. A. Ryzhov, N. N. Semenov, and M. N. Chemodanov, "Development the system of autonomous group management for heterogeneous surface and underwater unmanned vehicles", pp. 48-57 in 7th All-Russian Scientific and Technical Conference "Technical Problems of the Development of the World Ocean" (Vladivostok, Russia, 2017), Institute for Problems of Marine Technologies, Vladivostok, Russia, 2017. In Russian.

[Maggio et al. 2013] M. Maggio, E. Bini, G. Chasparis, and K.-E. Årzén, “A game-theoretic resource manager for RT applications", pp. 57-66 in Proceedings of the 25th Euromicro Conference on RealTime Systems (Paris, 2013), IEEE Computer Society, Los Alamitos, CA, 2013.

[Maslov 2005] V. P. Maslov, "Nonlinear averages in economics", Math. Notes 78:3-4 (2005), 347363.

[Tuphanov and Scherbatyuk 2015] I. E. Tuphanov and A. Ph. Scherbatyuk, "A centralized planner considering task spatial configuration for a group of marine vehicles: field test results", pp. 16791684 in 2015 IEEE/RSJ International Conference on Intelligent Robots and Systems (Hamburg, 2015), IEEE, Piscataway, NJ, 2015.

[Zaslavsky 2007] G. M. Zaslavsky, The physics of chaos in Hamiltonian systems, 2nd ed., Imperial College, London, 2007.

Received 9 Jan 2019. Revised 21 Apr 2019. Accepted 27 Jun 2019.

Mikhail A. GuZEv: guzev@iam.dvo.ru

GURAMI SH. TSITSIASHVILI: guram@iam.dvo.ru

MARINA A. OSIPOVA: mao1975@list.ru

Institute for Applied Mathematics, Far Eastern Branch, Russian Academy of Sciences, Vladivostok, Russia 
EDITORIAL BOARD

ANTONIO CARCATERRA

ERIC A. CARLEN

FRANCESCO DELL'ISOLA

RAFFAELE ESPOSITO

ALBERT FANNJIANG

Gilles A. FrancFort

Pierangelo Marcati

JEAN-JACQUES MARIGO

PETER A. MARKOWICH

MARTIN OSTOJA-STARZEWSKI

PIERRE SEPPECHER

DAVID J. STEIGMANN

PAUL STEINMANN

PierRe M. SuQueT

MANAGING EDITORS

MICOL AMAR

ANGELA MADEO

MARTIN OSTOJA-STARZEWSKI

\section{ADVISORY BOARD}

ADNAN AKAY

Holm AltenbaCH

MICOL AMAR

HARM ASKES

TEODOR ATANACKOVIĆ

VICTOR BERDICHEVSKY

GuY BouchitTÉ

ANDREA BRAIDES

ROBERTO CAMASSA

MAURO CARFORE

ERIC DARVE

FELIX DARVE

ANNA DE MASI

Gianpietro Del Piero

Emmanuele Di Benedetto

VICTOR A. EREMEYEV

BERNOLD FIEDLER

IRENE M. GAMBA

DAVID Y. GAO

SERGEy GaVRILYUK

Timothy J. HeAley

DOMINIQUE JEULIN

ROgER E. KHAYAT

CORRADO LATTANZIO

ROBERT P. LIPTON

ANGELO LUONGO

ANGEla MADEO

JUAN J. MANFREDI

CARlo MARCHIORO

ANIL MISRA

ROBERTO NATALINI

PATRIZIO NEFF

Thomas J. Pence

ANDREY PIATNITSKI

ERRICO PRESUTtI

MARIO PUlVIRENTI

Lucio Russo

Miguel A. F. SANJUAN

PATRICK SElvaduraI

MIROSLAV ŠILHAVÝ

GUIDO SWEERS

ANTOINETTE TORDESILLAS

LEV TRUSKINOVSKY

JUAN J. L. VELÁZQUEZ

VINCENZO VESPRI

ANGELO VULPIANI msp.org/memocs

Università di Roma "La Sapienza", Italia

Rutgers University, USA

(CO-CHAIR) Università di Roma "La Sapienza", Italia

(TREASURER) Università dell'Aquila, Italia

University of California at Davis, USA

(CO-CHAIR) Université Paris-Nord, France

Università dell' Aquila, Italy

École Polytechnique, France

DAMTP Cambridge, UK, and University of Vienna, Austria

(CHAIR MANAGING EDITOR) Univ. of Illinois at Urbana-Champaign, USA

Université du Sud Toulon-Var, France

University of California at Berkeley, USA

Universität Erlangen-Nürnberg, Germany

LMA CNRS Marseille, France

Università di Roma "La Sapienza", Italia

Université de Lyon-INSA (Institut National des Sciences Appliquées), France (CHAIR MANAGING EDITOR) Univ. of Illinois at Urbana-Champaign, USA

Carnegie Mellon University, USA, and Bilkent University, Turkey

Otto-von-Guericke-Universität Magdeburg, Germany

Università di Roma "La Sapienza”, Italia

University of Sheffield, UK

University of Novi Sad, Serbia

Wayne State University, USA

Université du Sud Toulon-Var, France

Università di Roma Tor Vergata, Italia

University of North Carolina at Chapel Hill, USA

Università di Pavia, Italia

Stanford University, USA

Institut Polytechnique de Grenoble, France

Università dell' Aquila, Italia

Università di Ferrara and International Research Center MEMOCS, Italia

Vanderbilt University, USA

Gdansk University of Technology, Poland

Freie Universität Berlin, Germany

University of Texas at Austin, USA

Federation University and Australian National University, Australia

Université Aix-Marseille, France

Cornell University, USA

École des Mines, France

University of Western Ontario, Canada

Università dell' Aquila, Italy

Louisiana State University, USA

Università dell'Aquila, Italia

Université de Lyon-INSA (Institut National des Sciences Appliquées), France University of Pittsburgh, USA

Università di Roma "La Sapienza", Italia

University of Kansas, USA

Istituto per le Applicazioni del Calcolo "M. Picone", Italy

Universität Duisburg-Essen, Germany

Michigan State University, USA

Narvik University College, Norway, Russia

Università di Roma Tor Vergata, Italy

Università di Roma “La Sapienza”, Italia

Università di Roma “Tor Vergata", Italia

Universidad Rey Juan Carlos, Madrid, Spain

McGill University, Canada

Academy of Sciences of the Czech Republic

Universität zu Köln, Germany

University of Melbourne, Australia

École Polytechnique, France

Bonn University, Germany

Università di Firenze, Italia

Università di Roma La Sapienza, Italia

MEMOCS (ISSN 2325-3444 electronic, 2326-7186 printed) is a journal of the International Research Center for the Mathematics and Mechanics of Complex Systems at the Università dell'Aquila, Italy.

Cover image: "Tangle” by $\odot$ John Horigan; produced using the Context Free program (contextfreeart.org).

PUBLISHED BY

7 mathematical sciences publishers

nonprofit scientific publishing

http://msp.org/

(C) 2019 Mathematical Sciences Publishers 
Mathematics and Mechanics of Complex Systems vol. 7 no. 2

A polynomial chaos expanded hybrid fuzzy-stochastic model for transversely fiber reinforced plastics

Eduard Penner, Ismail Caylak, Alex Dridger and Rolf Mahnken

Dynamic boundary conditions for membranes whose surface energy depends on the mean and Gaussian curvatures

Sergey Gavrilyuk and Henri Gouin Energy-based trajectory tracking and vibration control for multilink highly flexible manipulators

Ivan Giorgio and Dionisio Del Vescovo

A model of the proppant flowback: setup of the theoretical framework

Ksenia P. Frolova, Polina M. Grigoreva, Konstantin E.

Lezhnev and Grigoriy V. Paderin

The object detection by autonomous apparatus as a solution of the 189 Buffon needle problem

Mikhail A. Guzev, Gurami S. Tsitsiashvili and Marina A.

Osipova

MEMOCS is a journal of the International Research Center for the Mathematics and Mechanics of Complex Systems at the Università dell' Aquila, Italy.

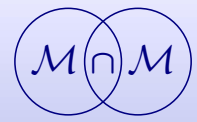

